Wright State University

CORE Scholar

Mechanical and Materials Engineering Faculty

Publications

Mechanical and Materials Engineering

2016

\title{
Silver Nanoparticles Supported on Carbon Nanotube Carpets: Influence of Surface Functionalization
}

\author{
Anil K. Karumuri \\ Dhawal P. Oswal \\ Heather A. Hostetler \\ Wright State University - Main Campus \\ Sharmila M. Mukhopadhyay \\ Wright State University - Main Campus, sharmila.mukhopadhyay@wright.edu
}

Follow this and additional works at: https://corescholar.libraries.wright.edu/mme

Part of the Materials Science and Engineering Commons, and the Mechanical Engineering Commons

\section{Repository Citation}

Karumuri, A. K., Oswal, D. P., Hostetler, H. A., \& Mukhopadhyay, S. M. (2016). Silver Nanoparticles

Supported on Carbon Nanotube Carpets: Influence of Surface Functionalization. Nanotechnology, 27 (14), 145603.

https://corescholar.libraries.wright.edu/mme/254

This Article is brought to you for free and open access by the Mechanical and Materials Engineering at CORE Scholar. It has been accepted for inclusion in Mechanical and Materials Engineering Faculty Publications by an authorized administrator of CORE Scholar. For more information, please contact library-corescholar@wright.edu. 


\title{
Silver nanoparticles supported on carbon nanotube carpets: influence of surface functionalization
}

\author{
Anil K Karumuri ${ }^{1}$, Dhawal P Oswal ${ }^{2}$, Heather A Hostetler ${ }^{2,3}$ and \\ Sharmila M Mukhopadhyay ${ }^{1}$ \\ ${ }^{1}$ Center for Nanoscale Multifunctional Materials, Wright State University, Dayton-45435, USA \\ ${ }^{2}$ Department of Biochemistry and Molecular Biology, Wright State University, Dayton—45435, USA \\ E-mail: smukhopa@wright.edu
}

Received 14 December 2015, revised 29 January 2016

Accepted for publication 4 February 2016

Published DD MM 2016

\begin{abstract}
The effectiveness of nanoparticle-based functional devices depends strongly on the surface morphology and area of the support. An emerging powerful approach of increasing the available surface area without decreasing strength or increasing bulk is to attach arrays of suitable nanotubes on the surface, and to attach the necessary nanoparticles to them. Earlier publications by this team have shown that carpet-like arrays of carbon nanotubes (CNT) can be successfully grown on a variety of larger carbon substrates such as graphite, foams and fabric, which offer hierarchical multiscale supporting architecture suitable for the attachment of silver nanoparticles (AgNPs). A limiting factor of pure CNT arrays in fluid-based applications is their hydrophobicity, which can reduce the percolation of an aqueous medium through individual nanotubes. Previous studies have demonstrated that the treatment of CNT carpets with dry (oxygen) plasma can induce reversible wettability, and treatment with wet (sol-gel) coating can impart permanent wettability. In this paper, we report the influence of such treatments on the attachment of AgNPs, and their effectiveness in water disinfection treatments. Both types of hydrophilic surface treatments show an increase in silver loading on the CNT carpets. Oxygenplasma treated surfaces (O-CNTs) show fine and densely packed AgNP, whereas silica-coated nanotubes (silica-CNTs) show uneven clusters of AgNPs. However, O-CNT surfaces lose their hydrophilicity during AgNP deposition, whereas silica-CNT surfaces remain hydrophilic. This difference significantly impacts the antibacterial effectiveness of these materials, as tested in simulated water containing Gram negative Escherichia coli (E. coli, JM109). AgNPs on silicacoated CNT substrates showed significantly higher reduction rates of E. coli compared to AgNPs on plasma-treated CNT substrates, despite the finer and better dispersed AgNP distribution in the latter. These results provide important insights into different aspects of surface modification approaches that can control the wettability of CNT carpets, and their applicability in water treatment applications.
\end{abstract}

Keywords: carbon nanotube carpets, surface wettability, silver nanoparticles, bacterial degradation with nanostructured solids, water reusability with nanomaterials

\section{Introduction}

3 deceased.

Nanoparticles (NPs) of noble metals such as silver offer several promising applications such as chemical-free 
disinfection devices, plasmonic sensors, and catalysts [1, 2]. The performance and targeted functionality of nanomaterialenhanced solid devices can depend heavily on the properties of the support and available surface area. Mukhopadhyay et al have developed an approach of attaching carpet-like arrays of carbon nanotubes (CNTs) onto porous carbon supports that results in ultra-high specific surface area (SSA) hierarchical substrates [3] suitable for supporting functional NPs. CNTs offer several advantages as NP supports and several earlier studies have discussed the influence of the physical and chemical nature of various support structures on advanced applications [4]. The CNT-enhanced substrates reported by our group have proved to be useful for several applications such as nanocomposite formation [5], surface adsorption [6], cell scaffolding [7], thermal management materials [8] in addition to support for metallic NPs [9-11].

However, an inherent property of pure, defect-free CNT arrays is that they tend to be super-hydrophobic due to the non-polar nature of pure graphene surfaces. This may be a limiting factor for both NP attachment using polar solvents and also for applications where the device needs to interact with water or other polar fluids. In such situations, fluid will not percolate well into the depths of the CNT carpet, hence making the 'effective surface area' of the device significantly lower than the actual surface area. Such devices will benefit if the nanotubes in the carpet are functionalized to be hydrophilic.

The surface functionalization of isolated nanotubes has been studied by many investigators with the idea of increasing their dispersion in solvents or polymer resins [12-15], and to promote NP growth [16-18]. Some studies have focused on modifying CNT arrays attached to flat model electronic substrates such as silicon [19, 20]. Common functionalization techniques involve CNT growth or treatment under a mild oxidizing environment or exposure to acids, organic groups or other liquid agents. Dry functionalization approaches include thermal or plasma treatments [19], carbon dioxide, UV/ozone radiation [14], or exposure to air/water mixed vapors at high temperatures [15]. When CNTs were grown under an oxygenrich environment, the CNT surface was enriched with hydroxyl groups which acted as activation sites for subsequent silver nanoparticle (AgNP) growth [16]. Similar observations were made $[17,18]$ when CNT carpets were treated under dry and/or wet oxidizing chemistries prior to AgNP growth. Similarly, non-oxidative methods such as grafting certain polymeric groups [21-23] were also seen to increase the AgNP growth and subsequent antibacterial activity. The success of wettability alteration and durability resulting from each method should depend on interatomic bonds created between the graphitic nanotubes and surface functional groups, but is not clearly addressed in all cases. For instance, it was indicated that some oxidative functionalization methods lead to dynamic wettability [24], but the wettability of polymer-functionalized CNT used for AgNP growth was not clear [21-23].

Our group has investigated the surface modification of CNT carpets on both flat and porous carbon substrates. It was reported earlier [25] that short-term exposure to microwave plasma can impart reversible hydrophilic properties on the surface that can be easily reversed by heating. In fact, the contact angles (CAs) could be cycled between $165 \pm 2^{\circ}$ to less than $10^{\circ}$ degrees by repeated cycles of plasma treatment and air annealing. On the other hand, permanent surface hydrophilic behavior could be imparted to these CNT carpets by coating them with an inorganic silica layer using a modified sol-gel method [25]. The latter method resulted in a permanent decrease in the CA from 160 to $35^{\circ}$.

In this paper, we report the influence of these surface modification processes on the growth and anti-bacterial behavior of AgNPs attached to CNT-coated surfaces. AgNPs are deposited by the in situ reduction of silver salts using suitable reducing and capping agents. This process involves treatment of the surface with water-based precursors. It is therefore expected that the enhanced wettability of the CNTs induced by surface treatments in this study should influence how precursors percolate through the CNT carpets, and affect the overall AgNP growth. In this study, we have compared untreated CNT carpets with oxygen-plasma-treated and silicacoated carpets. The size distribution and overall loading densities of the AgNPs have been analyzed using high-resolution microstructures and image analysis. The wettability of the surfaces before and after AgNP growth has been monitored using CA measurements. Finally, the bacteria degradation rates of the AgNP-deposited structures have been compared.

\section{Materials and methods}

\subsection{Materials}

2.1.1. Selected supports. Two kinds of carbon support structures were used in this study. Highly oriented pyrolytic graphite (HOPG) [HOPG ZYH grade provided by Molecular Imaging] was used as a model flat substrate suitable for indepth characterization of NPs and CA measurements. Reticulated vitreous carbon (RVC) foam [grade-80 pores per inch provided by Ultramet Inc.] was selected as the substrate for testing antibacterial degradation and for deployment in water purification devices.

\subsection{Methods}

2.2.1. Attachment of carbon nanotube (CNT) carpets on selected substrates. CNTs were grafted onto selected support structures using a two-step method discussed in earlier publications [3]. This involves plasma treatment to create a nanolayer of Si-O groups on the surface followed by chemical vapor deposition (CVD) using the floating catalyst technique.

2.2.2. Surface modifications for wettability. Two surface modification techniques were investigated in this study. The first is dry plasma etching with oxygen gas, which involves short duration ( $5 \mathrm{~s}$ ) exposure of CNT-attached substrates to oxygen microwave plasma. The second involves a sol-gel 

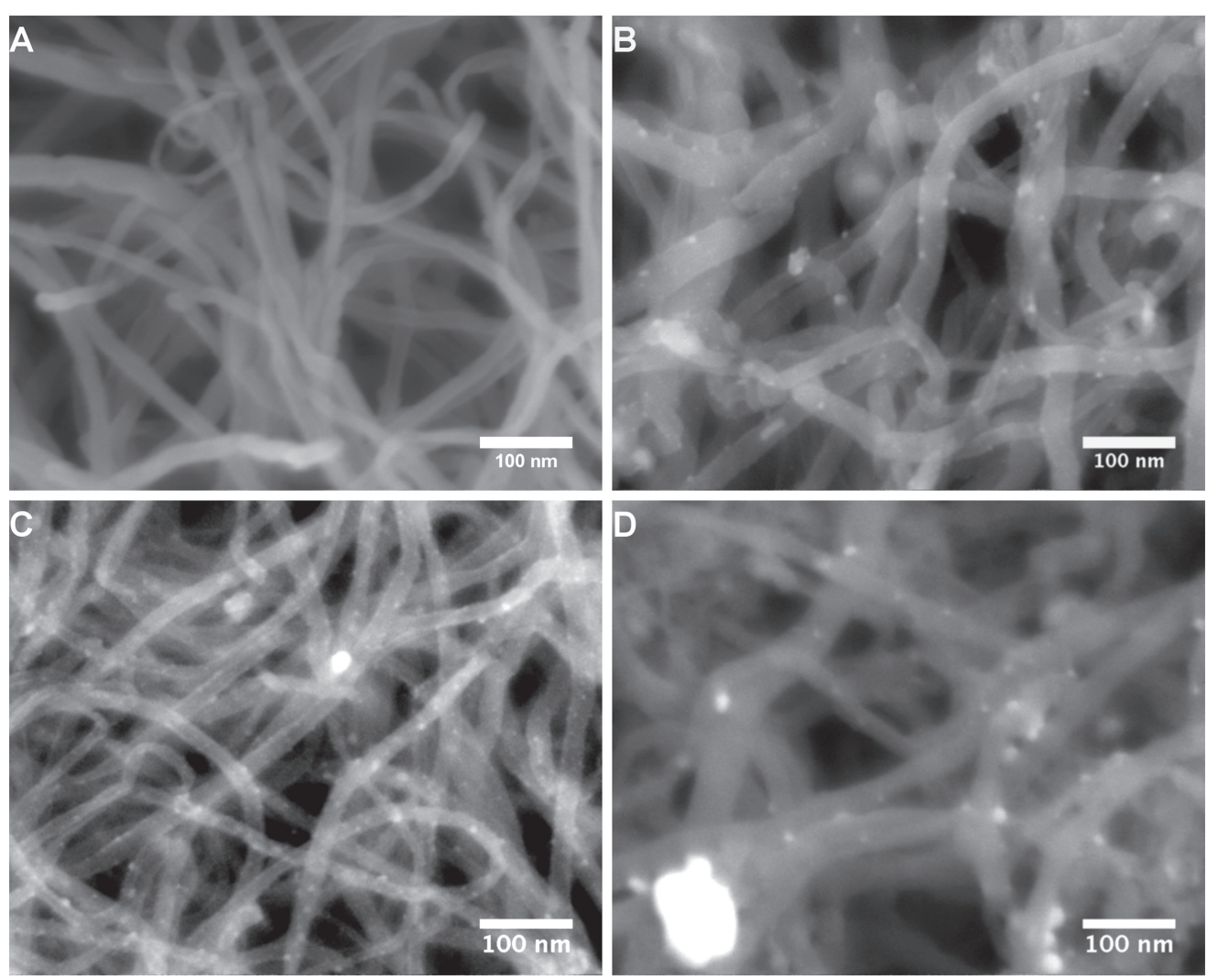

Figure 1. Microstructures of CNT-HOPG (A), AgNP-deposited CNT-HOPG (B) O-CNT-HOPG. (C), and silica-CNT-HOPG (D).

silica coating, created by dipping and drying the CNT-carpets in a silica precursor solution, followed by controlled heating. The details of both treatments have been reported in an earlier publication [25].

2.2.3. Synthesis of AgNP on support structures. For the synthesis of AgNPs, silver nitrate was used as the metal precursor $\left[\mathrm{AgNO}_{3}\right.$ (Sigma Aldrich; 99.99\%)], dimethyl sulfoxide was the selected reducing agent [26] [DMSO (Sigma Aldrich; 99.5\%)], and trisodium citrate was used as the stabilizing agent [26] $\left(\mathrm{Na}_{3} \mathrm{C}_{6} \mathrm{H}_{5} \mathrm{O}_{7}, \mathrm{MP}\right.$ bio; 99\%). The overall process is discussed in an earlier publication [10] and involves two steps: $\mathrm{AgNO}_{3}$ deposition from an aqueous solution followed by a reduction in the presence of a sodium citrate-DMSO solution [27]. The only change made in this study compared to earlier ones [10] is that here, the molarity $\mathrm{AgNO}_{3}$ precursor solution was increased to $0.72 \mathrm{M}$ compared to about $0.24 \mathrm{M}$ in most reported studies.

\section{Characterization methods}

Microstructure and spectroscopic characterization of these materials at every step was performed using field emission scanning electron microscopy (FESEM), x-ray photoelectron spectroscopy (XPS), and x-ray diffraction (XRD).
Table 1. Percentage weight of elements presented on the surface (EDS data averaged over five scans).

\begin{tabular}{lccc}
\hline Substrate & $\begin{array}{c}\text { AgNP- } \\
\text { CNT-HOPG }\end{array}$ & $\begin{array}{c}\text { AgNP-O- } \\
\text { CNT-HOPG }\end{array}$ & $\begin{array}{c}\text { AgNP-silica- } \\
\text { CNT-HOPG }\end{array}$ \\
\hline CK & 78.25 & 72.73 & 64.27 \\
OK & 6.89 & 10.56 & 12.39 \\
SiK & 7.56 & 8.58 & 12.18 \\
AgL & 1.74 & 4.59 & 6.6 \\
FeL & 5.54 & 3.52 & 4.56 \\
\hline
\end{tabular}

\subsection{Imaging and analysis}

Detailed macro, micro and nanoscale structural analyses of CNT-grafted carbon structures were performed using FESEM (JEOL 7401F). Both secondary (SE) and backscattered (BE) imaging modes were used for structural analysis. The particle size distributions of the AgNPs were measured from the images using the Scandium imaging software.

\subsection{Antibacterial performance test}

3.2.1. Influent water preparation. E. coli, $K 12$ derivative, was selected as a test microbe due to its role as an indicator of fecal contamination in water. E. coli culture was prepared using a standard method and discussed in earlier reports [1]. The concentration of prepared influent water (E. coli dispersed pure water) was $10^{3} \mathrm{CFU} \mathrm{ml}^{-1}$ and used for testing. The antibacterial performance of the prepared 

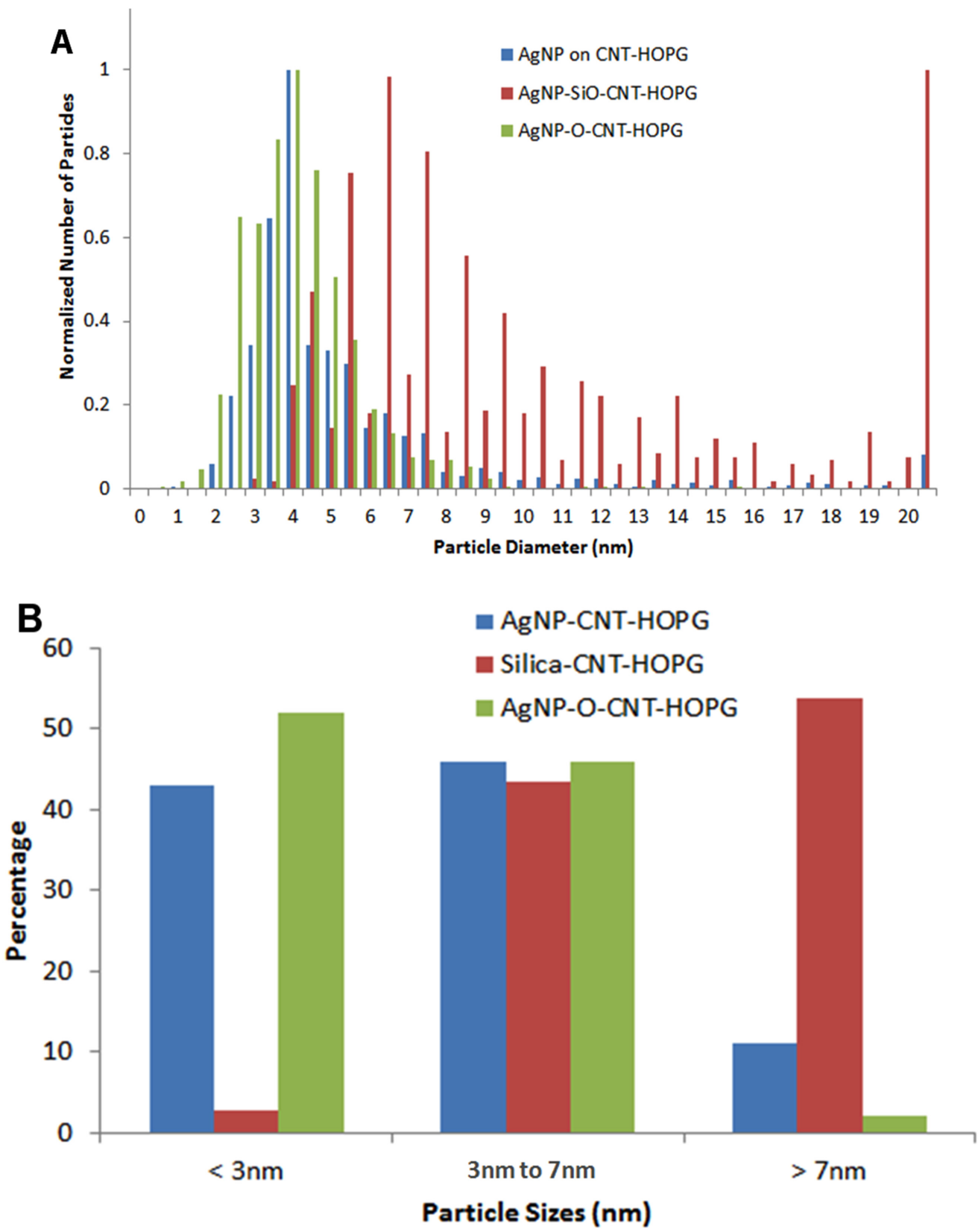

Figure 2. Histogram of particle size distribution (A); Summarized histogram of size distribution.(B).

Table 2. Summary of NP size and deposition densities.

\begin{tabular}{lcccrr}
\hline Substrate & No. of particles considered & Ag wt\% (from EDS) & Average particle size $\left(R_{p}\right)$ & $N_{C N T}(/ \mu \mathrm{m})$ & PPA $\left(/ \mu \mathrm{m}^{2}\right)$ \\
\hline CNT-HOPG & 848 & 1.74 & $5.5 \pm 4 \mathrm{~nm}$ & $11.2 \pm 2$ & $\sim 6.2 \times 10^{4}$ \\
O-CNT-HOPG & 995 & 4.59 & $4.1 \pm 2 \mathrm{~nm}$ & $31 \pm 7$ & $\sim 1.7 \times 10^{4}$ \\
Silica-CNT-HOPG & 486 & 6.6 & $3-180 \mathrm{~nm}$ (median $8.4 \mathrm{~nm})$ & $8.6 \pm 3$ & $\sim 4.8 \times 10^{4}$ \\
\hline
\end{tabular}

samples $[11.5 \mathrm{~mm}$ (diameter) $\times 4 \mathrm{~mm}$ (thickness) $]$ was tested in two modes-rotation and filtration.

3.2.2. Antibacterial performance via rotation. In the case of the rotation mode, the sample was taped to the wall of a glass jar filled with $500 \mathrm{ml}$ influent water. The jar was tumbled at a speed of $60 \mathrm{RPM}$ for $40 \mathrm{~min}$. $50 \mu \mathrm{l}$ aliquots of the water sample were acquired at 0,5,10,20, and 40 min and streaked on agar plates.

3.2.3. Antibacterial performance via filtration. In the case of the filtration test, influent water was passed through a cartridge fitted with the foam sample to be tested. A schematic representation of the experimental setup was 

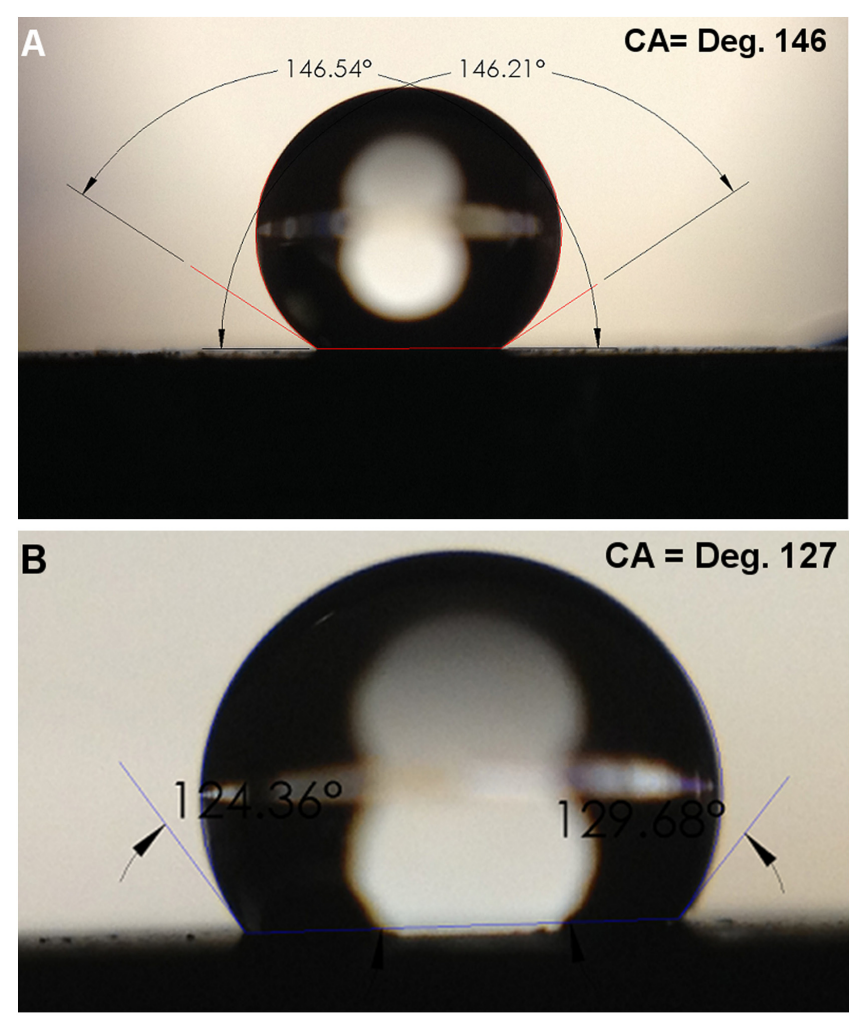

C

$C A=$ Deg. 31

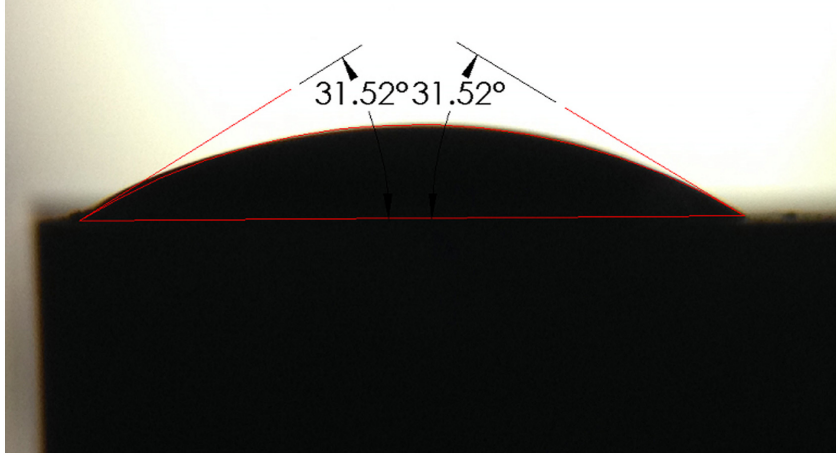

Figure 3. Surface wettability of various AgNP-deposited surfacemodified surfaces; AgNP-CNT-HOPG (A), AgNP-O-CNT-HOPG (B), and AgNP-silica-CNT-HOPG (C).

shown in an earlier publication [1]. $50 \mu \mathrm{l}$ aliquots of the water sample were acquired at the start and the end of each filtration step and streaked on three agar plates in order to measure the change in $E$. coli activity with each pass.

The agar plates were incubated at $37^{\circ} \mathrm{C}$ for $20 \mathrm{~h}$ and the bacteria colonies were counted visually. The results are reported as a fraction of $E$. coli survival (colonies formed from the water sampled after the treatment over the initial E. coli load).

\section{Results and discussion}

\subsection{Influence of CNT functionalization on AgNP distribution}

For this study, CNT-HOPG was taken as the control substrate. This was functionalized by two techniques described earlier: (i) oxygen-plasma treatment (samples labeled as O-CNT-HOPG) and (ii) silica coating using a sol-gel method (samples labeled as silica-CNT-HOPG). AgNPs were deposited on three types of supports; CNT-HOPG, O-CNTHOPG, and silica-CNT-HOPG, as discussed in section 2.2. Sample SEM images of the resulting AgNP distribution on various surfaces are shown in figure 1 and a statistical average over a large number of analysis spots is summarized in tables 1 and 2. The following key observations are noted:

\section{(i) Overall elemental composition by EDS}

Overall elemental quantification was performed by energy dispersive spectroscopy (EDS) using @7 kV accelerating voltage from these samples. The results are tabulated in table 1. It must be noted here that EDS gives approximate percentage compositions averaged over the top of several microns of sample depth. Hence, for the hierarchical surfaces involving uneven morphology comprising tens of microns of CNT carpet, the absolute values of EDS data are not expected to be accurate. What is important to note is how the values in identical nanocarpet morphology change with each processing method. All samples show iron signal, remnants from the CVD process where iron nanoparticles (FeNP) catalyze CNT growth. These have been carefully measured and reported in an earlier study [11], indicating that most of the FeNPs in clean CNT growth conditions are confined inside the nanotubes and in the deeper regions of the CNT carpet, and hence do not interfere with the functionality of the AgNPs. Silicon signal increases with sol-gel silica deposition, as expected. It must be noted that the oxygen content is seen to increase with both treatments and is significantly higher than that of surface-adsorbed adventitious oxygen expected in untreated samples exposed to air. The comparative EDS data indicate that, under identical deposition conditions, both surface functionalization methods increase surface oxygen, and also significantly increase the total uptake of silver on the CNT carpets. The silica-CNT-HOPG sample has the highest amount of total silver, followed by O-CNT-HOPG, both having more than twice the silver uptake of untreated CNT-HOPG.

\section{(ii) Nanoparticle distribution}

NP distributions on the three samples were studied using size distribution analysis and areal density calculations. It was shown in our earlier report that the CNT-HOPG sample

Table 3. Contact angle before and after AgNP growth.

\begin{tabular}{lrlc}
\hline Substrate & CNT-HOPG & O-CNT-HOPG & Silica-CNT-HOPG \\
\hline Before AgNP growth [25] & $162.5 \pm 2^{\circ}$ & $\leqslant 10^{\circ}$ & $45 \pm 5^{\circ}$ \\
After AgNP growth & $146^{\circ} \pm 2^{\circ}$ & $127 \pm 2^{\circ}$ & $31^{\circ} \pm 1^{\circ}$ \\
\hline
\end{tabular}



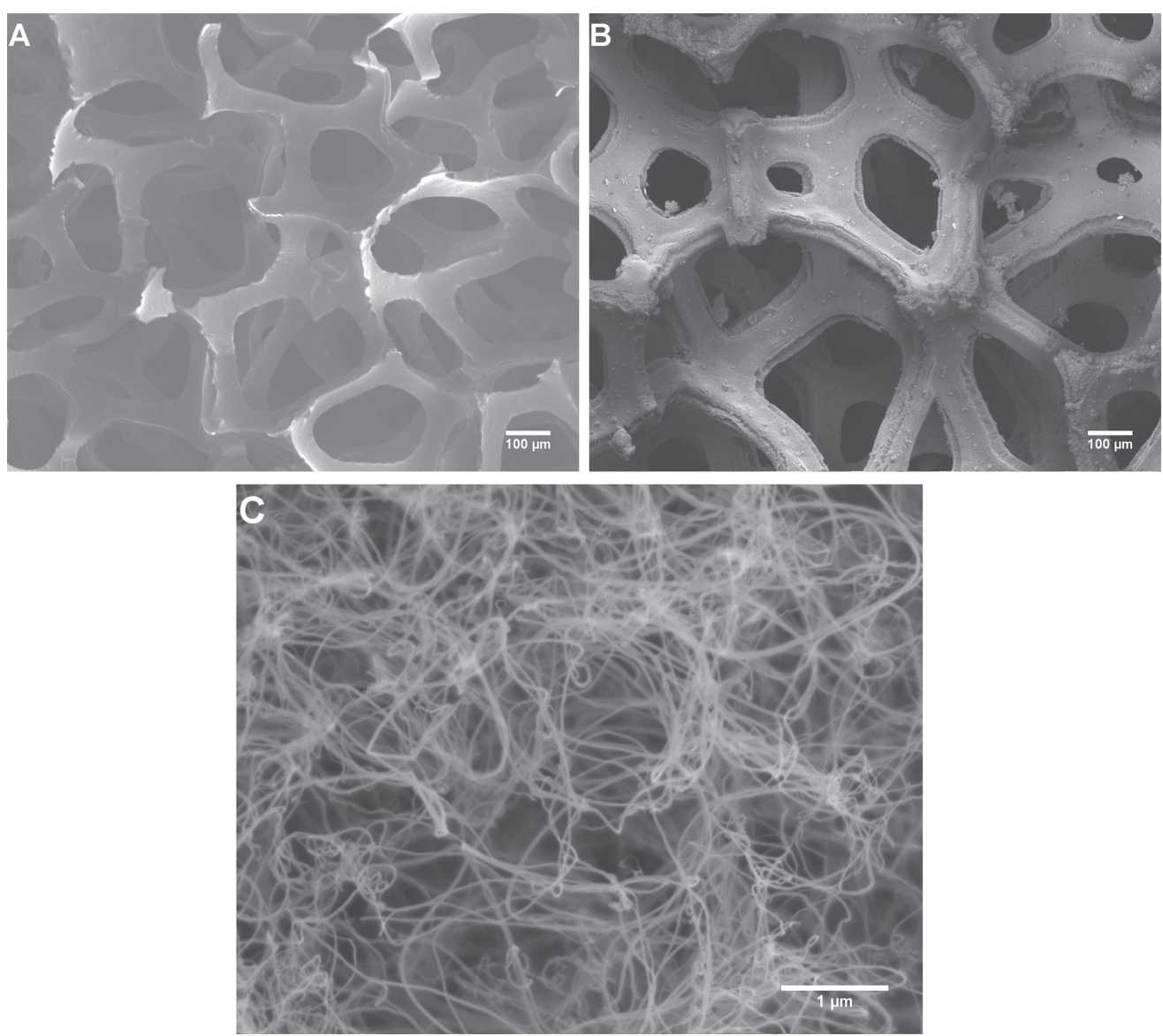

Figure 4. RVC foam microstructure before (A) and after CNT grafting (B). High-resolution image of CNT grown on cell walls (C).

contained a small but detectable amount of metallic NPs of iron [11] even prior to AgNP deposition. However, in an aerial SEM image, these were reported to be a small fraction (less than one order of magnitude) of the total NPs and therefore have negligible influence on the current AgNP analysis. Moreover, these are inherent to the CVD process and expected to be identical for all three samples, and hence do not interfere with any changes in AgNP distribution reported in this study. Multiple microstructures from each type of sample were analyzed to obtain a statistical analysis of particle size distribution, average particle size, and total particle density, tabulated in table 2 .

A histogram of particle size distribution from all three samples is shown in figure 2. A summarized version of this observation is presented in figure 2(B) with NPs categorized into three discrete bins; less than $3 \mathrm{~nm}, 3$ to $7 \mathrm{~nm}$, and greater than $7 \mathrm{~nm}$. In the case of AgNPs grown on CNT-HOPG, the percentages of particles falling into the aforementioned regions are $42.9,45.9$, and $11 \%$ respectively. On O-CNTHOPG the corresponding percentages are 52, 45.8 and $2.1 \%$ respectively, indicating a noticeable preference for smaller NP size. This can be attributed to isolated nucleation sites resulting from oxygen-containing functional groups (C-O/ $\mathrm{OH})$ attached on CNT confirmed by XPS data reported previously [16]. It is expected that such sites will immobilize the nucleating metal clusters, preventing their agglomeration into larger particles. Similar observations on isolated nanotubes were reported by other researchers [16] who concluded that oxygen-functionalized CNT stabilizes the NPs from agglomeration, resulting in finer particles compared to the NPs grown on pristine CNT.

Silica-CNT-HOPG surfaces show a completely different behavior. Here, size distribution does not follow any statistical distribution, and spreads across a wide range. The fraction of finer NPs ( $4 \mathrm{~nm}$ or less) is considerably lower than in the other two samples. Moreover, a significant percentage (approximately 12\%) of AgNPs are larger than $20 \mathrm{~nm}$, and many are clustered together to form localized AgNP patches. The clustering of NPs on the silica-CNT-HOPG surface may be due to a continuous hydrophilic layer on the nanotube surfaces that may hold a thicker layer of precursor components as a continuous coating, which makes it easier for them to agglomerate during reduction treatment and metal formation. In addition, the sol-gel film thicknesses on these high aspect ratio features are expected to be non-uniform, and local variations in precursor thickness can result in local changes in precursor adsorption and nucleation/growth conditions for NPs.

(iii)Density of nanoparticles (Number of particles per area (PPA)) 

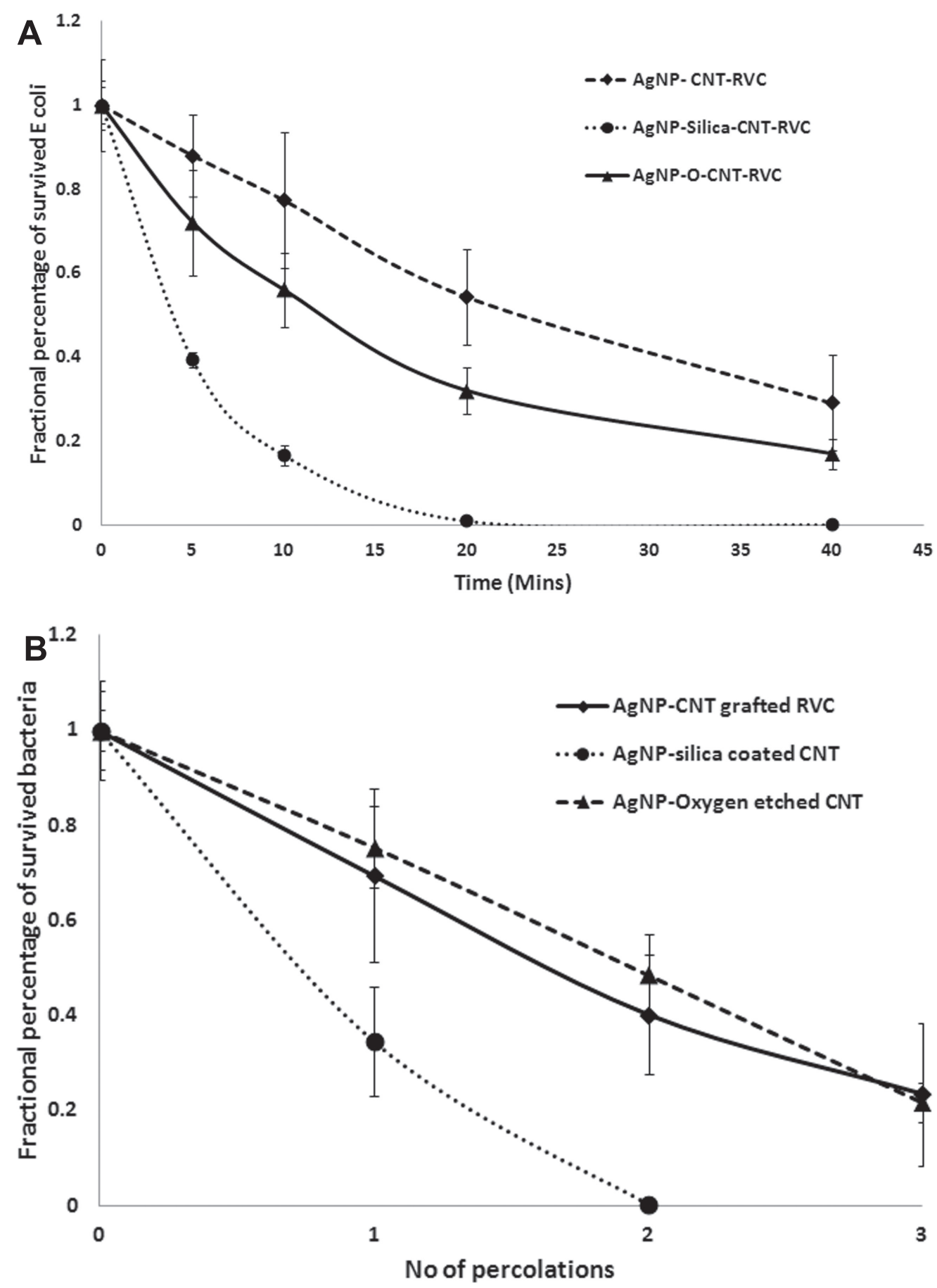

Figure 5. Antibacterial test results; rotation (A) and filtration (B).

The areal density of NPs (\# of particles per area of base substrate (PPA)) can be estimated from microstructural analysis of the average NP count per unit length of nanotube and using the formula below.

$$
\begin{aligned}
P P A & =\text { Arial density of } N P\left(\frac{1}{\mu \mathrm{m}^{2}}\right) \\
& =N P_{C N T}\left(\frac{1}{\mu \mathrm{m}}\right) \times L_{C N T} \times C N T_{A}\left(1 / \mu \mathrm{m}^{2}\right)
\end{aligned}
$$

Here, $\left(N P_{C N T}\right)$ is \# of NPs per unit length of CNT, $\left(L_{C N T}\right)$ is the average length of the CNT carpet, and $\left(\mathrm{CNT}_{\mathrm{A}}\right)$ is the number of CNTs per unit area of the substrate. Previous studies of CNT carpet growth on the same grade of HOPG substrate under identical conditions [28] have reported that the best estimates for $\left(L_{C N T}\right)$ and $\left(C N T_{A}\right)$ using this deposition method are about $20 \mu \mathrm{m}$ and $280 \mu \mathrm{m}^{-2}$, which match well with direct physical property measurements [6].

In this study, detailed microstructural analysis was performed to get the best possible estimate for $\mathrm{NP}_{C N T}$, the number of NPs per unit length of CNT. This was performed 

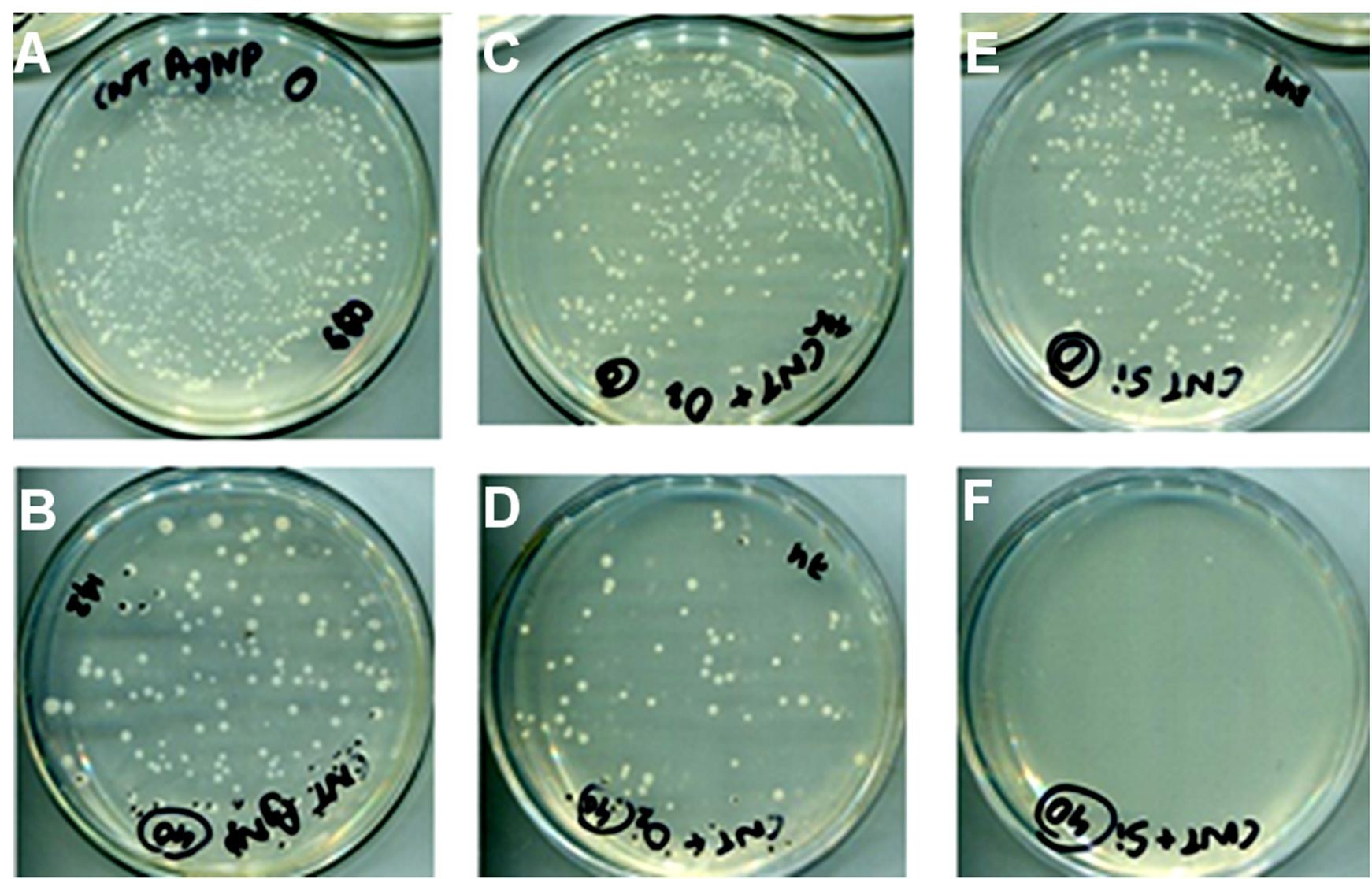

Figure 6. Active E. coli regrown on agar plates from the rotation test; AgNP-CNT-RVC@ 0 min (A), 40 min (B), AgNP-O-CNT-RVC @ 0 min (C), 40 min (D), and AgNP-silica-CNT-RVC @ 0 min (E), 40 min (F).

on nanotubes whose lengths were fully within focus of the scanning electron images. That is, unlike counting all visible particles as was done for size-distribution analysis, this study counted only the particles that could be clearly verified as being attached to the nanotube in focus, as a fraction of the CNT length that was in focus. The statistical summary of these values, averaged over a large number of images, is summarized in table 2 .

This analysis shows that $\mathrm{NP}_{C N T}$ estimates of CNTHOPG, O-CNT-HOPG and silica-AgNP-CNT-HOPG are $11.2 \pm 2 \mu \mathrm{m}^{-1}, 31 \pm 7 \mu \mathrm{m}^{-1}$, and $8.6 \pm 3 \mu \mathrm{m}^{-1}$ respectively. From this analysis, it is clear that plasma treatment of CNTs leads to finer nanoparticles having about three times higher linear density (NP per length of CNT) compared to untreated CNTs. On the other hand, silica coating, which clearly increases coarsening and cluster formation, lowers the numerical count of individual NPs. It is possible that in these latter samples, multiple NPs in a cluster are counted as one, which will lead to underestimation of $\mathrm{NP}_{C N T}$. It is also noted that these NPs are significantly larger in size, and have a wider distribution, which disallows statistical estimates. The areal density of the NPs (PPA) was calculated from substituting $N P_{C N T}, L_{C N T}$, and $C N T_{A}$ values in the above equation and are indicated in table 2.

(iv) Contact angle measurement
The CAs of water on these samples were measured before and after AgNP deposition using a standard labassembled goniometer as described in earlier studies [25]. Figure 3 shows sample images of a water droplet on samples after AgNP deposition, and results are tabulated in table 3. Prior to AgNP deposition, the CAs of the CNT carpets were measured to be $162.5 \pm 2^{\circ},<10^{\circ}$ and $45 \pm 5^{\circ}$ respectively, the same as reported in an earlier publication [25]. It is clear that an untreated CNT surface is super-hydrophobic, but becomes hydrophilic with both these treatments. However, after AgNP attachment, the CA changes differently in each case. The AgNP deposition step causes a slight decrease in the CAs for the CNT-HOPG and silica-CNT-HOPG samples, probably due to traces of residual impurities from the liquidphase precursors. However, the plasma-treated O-CNT sample (that was strongly hydrophilic prior to $\mathrm{Ag}$ deposition) showed a very significant increase in the CA after AgNP deposition, and became hydrophobic.

This is an important observation, because the CA is an indication of surface morphology combined with chemical functional groups. Surface-functional bonds and related CA changes caused by oxygen plasma were reported earlier to be temporary and could be reversed by $110^{\circ} \mathrm{C}$ heat treatments [25]. This study indicates that even the process of AgNP deposition, which involves exposure to a precursor solution combined with very gentle heat treatment $\left(60^{\circ} \mathrm{C}\right)$, can reverse the wettability of plasma-oxygen treated CNT carpet to a 

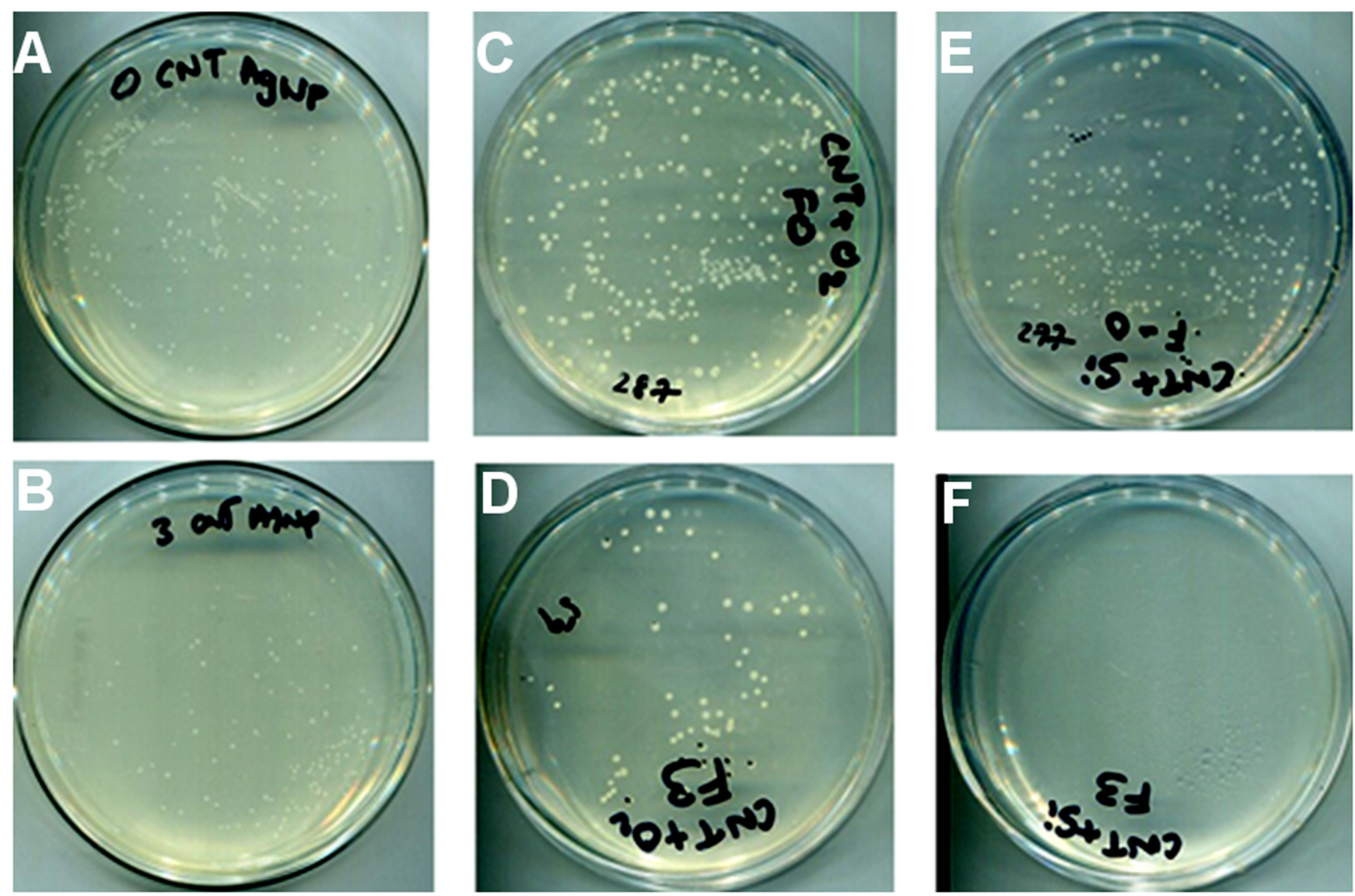

Figure 7. Active E. coli regrown on agar plates from the filtration test; AgNP-CNT-RVC @ 0 pass (A), 3rd pass 40 min (B), AgNP-O-CNTRVC @ 0 pass (C), 3rd pass(D), and AgNP-silica-CNT-RVC @ 0 pass (E), 3rd pass (F).

significant extent, if not fully. This property needs to be taken into consideration before this surface modification method is used for device development.

\subsection{Antibacterial performance test}

In order to test the antibacterial effectiveness, AgNP was attached to CNT carpets grown on RVC foam as described earlier. Figure 4 shows an image of a CNT carpet on RVC foam having $97 \%$ porosity. These structures offer several important advantages: The interconnected micro-sized pores of the RVC provide necessary structural integrity, strength, and flow channels for the fluid. The CNT carpets on RVC foam increases their surface area by $2-3$ orders of magnitude and enable higher NP deposition densities.

Three porous materials having identical morphology, but different surface treatments (hence different wettability and AgNP distribution) were compared under identical testing conditions: CNT grown RVC foam (CNT-RVC not treated), plasma-oxygen treated CNT-RVC (O-CNT-RVC) and silicacoated CNT-RVC (silica-CNT-RVC). The antibacterial performance of each sample was tested in two modes as described previously.

In the rotation test, the fraction of $E$. coli that was deactivated by AgNP-CNT-RVC, O-CNT-RVC and silicaCNT-RVC in 40 min was about 71,79 and $100 \%$ respectively (figure 5(A)). Despite the significantly higher (over 2.5 times or $250 \%$ ) silver content and AgNP deposition density (tables 1 and 2), the bacterial deactivation rates of AgNP-OCNT-RVC were very similar to the AgNP-CNT-RVC sample. In contrast, AgNP-silica-CNT-RVC, which has a higher overall silver but lower NP count, has complete deactivation of $E$. coli $(100 \%)$ in a much shorter span of time $(20 \mathrm{~min}$; figure $5(\mathrm{~A})$ ). This can clearly be attributed to the increased hydrophilic behavior of the sol-gel silica-coated sample that was retained even after silver deposition.

A very similar trend was seen in the filtration testing mode (figure 5(B)). NPs deposited on both CNT-RVC and O-CNT-RVC brought about a $76 \%$ decrease in the bacterial load in a total of three percolations, whereas AgNPs deposited on silica-CNT-RVC completely deactivated the bacterial load $(100 \%)$ in only two percolations (figure 5(B)). Samples of E. coli cells grown on agar plates at different water collection time-points in the two test modes were shown in figures 6 and 7 respectively. Table 4 summarizes the E. coli deactivation rates observed.

Bactericidal properties of the AgNP depends on many factors such as size [29], shape [30] total AgNP surface area available, and particle-cell contact [31]. It has been reported that the bactericidal effect increases with decreasing particle size [29] while the platelet-like NPs with a (111) lattice plane as the basal plane displayed the strongest biocidal action 
Table 4. Percentage of antibacterial efficiency with surface modifications (averaged over nine measurement points or three samples).

\begin{tabular}{lclll}
\hline Sample & $\begin{array}{c}\text { Efficiency in rotation @ } \\
20 \mathrm{~min}\end{array}$ & $\begin{array}{l}\text { Improvement over AgNP-CNT-RVC in } \\
\text { rotation }\end{array}$ & $\begin{array}{l}\text { Efficiency in filtration @ two } \\
\text { percolations }\end{array}$ \\
\hline AgNP-CNT-RVC & $71 \%$ & Control & $54 \%$ & control \\
AgNP-O-CNT-RVC & $79 \%$ & About $11 \%$ & $59 \%$ & about $9 \%$ \\
AgNP-silica- & $100 \%$ & About $40 \%$ & $100 \%$ & about $85 \%$ \\
CNT-RVC & & & & \\
\hline
\end{tabular}


compared to spherical or rod-shaped NPs. In addition, a recent study by Bondarenko et al [31] demonstrated that the direct contact between the bacterial cell and AgNP enhanced the toxicity of the AgNP.

From the AgNP distribution data shown in table 2, it is expected that the silver activity should be significantly higher for AgNP-O-CNT-RVC compared to that of AgNP-CNT (an almost threefold increase in areal density of AgNPs). Despite that, the increase in the bacteria degradation rate is small (only 9-11\%). On the other hand, the AgNP-silica-CNT sample shows significantly higher rates of bacteria removal. This can be attributed to water infiltration through the CNT carpets. It must be noted that the surface wettability is highest

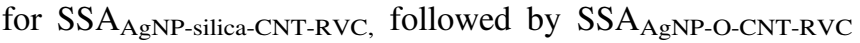
and lowest for SSA $\mathrm{AgNP}-\mathrm{CNT}-\mathrm{RVC}_{\mathrm{C}}$ as seen in figure 3 . This is the same order of bacteria removal rates. This indicates that the effectiveness of AgNP in bacterial degradation may depend significantly on the hydrophilicity of the nanotubes, which allows direct contact between the AgNP surfaces and microbes in water. This finding supports earlier publications [22] that point to the importance of Ag-bacteria physical contact in biocidal activity. The overall antibacterial efficiency of AgNP-silica-CNT-RVC far outperforms that of AgNP-O-CNT-RVC and AgNP-CNT-RVC. From these results, one can conclude that while improving the wettability of CNT carpets prior to NP functionalization can increase particle deposition density significantly, the antibacterial efficiency of the AgNP-containing solids will depend more significantly on the final wettability of the CNT carpets after final processing (deposition of AgNP in this case).

\section{Summary}

In this investigation, AgNPs were deposited on carbon nanotube carpets functionalized using two kinds of surface modification techniques: oxygen-plasma treatment and liquidphase silica coating. The influence of surface modification on AgNP growth, distribution, and deposition densities was studied using electron microscopy and image analysis. Results indicate that AgNP grown on plasma-oxygen-treated nanotubes (O-CNT-HOPG) yielded finer NPs, narrower size distribution, and over $250 \%$ higher particle densities compared to AgNP deposited on untreated CNT-HOPG. However, the AgNP-O-CNT-HOPG surface could not retain its wettability after the AgNP deposition step due to possible loss of hydrophilic groups from the surface during the silver deposition step. On the other hand, the AgNP-silica-CNTHOPG surface retained its higher wettability post AgNP deposition due to the more durable (permanent) silica coating.

The influence of the AgNP distribution and wettability of these structures on antibacterial effectiveness was studied using CNT carpets on RVC supports. Two different percolation modes (filtration and rotation) were tested. In both test modes, AgNP grown on silica-CNT-RVC showed the highest E. coli deactivation rates compared to not just the AgNPCNT-RVC sample, but the AgNP-O-CNT-RVC sample that had a higher density of AgNPs. This indicates that, in order to use these structures for the degradation of water-borne bacteria, in addition to the quantity of AgNPs, the CNT wettability and overall infiltration of water through the individual CNTs should be taken into consideration. These results provide a deeper understanding of crucial issues related to future design of fluid treatment and other devices using metallic NPs on nanotube carpets.

\section{Acknowledgments}

Financial support from the Ohio Third Frontier Program, Environmental Protection Agency, NSF-CBET (1449582) and Wright State University PhD fellowship is acknowledged. The facilities used were funded by the NSF-MRI award and Ohio Board of Regents. The authors are grateful to Ultramet Inc. for the generous supply of RVC foams.

\section{References}

[1] Karumuri A K, Oswal D P, Hostetler H A and Mukhopadhyay S M 2013 Silver nanoparticles attached to porous carbon substrates: robust materials for chemical-free water disinfection Mater. Lett. 109 83-7

[2] Krutyakov Y A, Kudrinskiy A A, Olenin A Y and Lisichkin G V 2008 Synthesis and properties of silver nanoparticles: advances and prospects Russ. Chem. Rev. 77 233-57

[3] Mukhopadhyay S M, Karumuri A and Barney I T 2009 Hierarchical nanostructures by nanotube grafting on porous cellular surfaces J. Phys. D. Appl. Phys. 42195503

[4] Saha A, Jiang C and Martí A A 2014 Carbon nanotube networks on different platforms Carbon NY 79 1-18

[5] Mukhopadhyay S M and Karumuri A K 2010 Nanotube attachment for prevention of interfacial delamination J. Phys. D. Appl. Phys. 43365301

[6] Vijwani H, Nadagouda M N, Namboodiri V and Mukhopadhyay S M 2015 Hierarchical hybrid carbon nanostructures as robust and reusable adsorbents: kinetic studies with model dye compound Chem. Eng. J. 268 197-207

[7] Maurer E, Hussain S and Mukhopadhyay S M 2011 Cell growth in a porous microcellular structure: influence of surface modification and nanostructures Nanosci. Nanotechnol. Lett. 3 110-3

[8] Barney I T, Ganguli S, Roy A K and S M M 2012 Improved thermal response in encapsulated phase change materials by nanotube attachment on encapsulating solid J. Nanotechnol. Eng. Med.

[9] Vijwani H and Mukhopadhyay S M 2012 Palladium nanoparticles on hierarchical carbon surfaces: a new architecture for robust nano-catalysts Appl. Surf. Sci. 263 712-21

[10] Vijwani H, Agrawal A and Mukhopadhyay S M 2012 Dechlorination of environmental contaminants using a hybrid nanocatalyst: palladium nanoparticles supported on hierarchical carbon nanostructures J. Nanotechnol. 2012

[11] Karumuri A K, Maleszewski A A, Oswal D P, Hostetler H A and Mukhopadhyay S M 2014 Fabrication and characterization of antibacterial nanoparticles supported on hierarchical hybrid substrates J. Nanoparticle Res. 162346 
[12] Hirsch A, Vostrowsky O, Chemie O and Erlangen-nürnberg U 2005 Functionalization of carbon nanotubes Top. Curr. Chem. 245 193-237

[13] Jiang C, Saha A and Martí A A 2015 Carbon nanotubides: an alternative for dispersion, functionalization and composites fabrication Nanoscale 7 15037-45

[14] Sham M-L and Kim J-K 2006 Surface functionalities of multiwall carbon nanotubes after UV/Ozone and TETA treatments Carbon NY $\mathbf{4 4}$ 768-77

[15] Liu J and Gong G 2012 Water oxidation of multi-walled carbon nanotubes under moderate industrial condition 2012 12th IEEE Int. Conf. Nanotechnol. pp 1-4

[16] Jiang H, Zhu L, Moon K and Wong C P 2007 The preparation of stable metal nanoparticles on carbon nanotubes whose surfaces were modified during production Carbon NY 45 655-61

[17] Dinh N X, Van Quy N, Huy T Q and Le A-T 2015 Decoration of silver nanoparticles on multiwalled carbon nanotubes: antibacterial mechanism and ultrastructural analysis J. Nanomater. 2015 1-11

[18] Prodana M, Ionita D, Ungureanu C, Bojin D and Demetrescu I 2011 Enhancing antibacterial effect of multiwalled carbon nanotubes using silver nanoparticles Microscopy 6 549-56

[19] Li P, Lim X, Zhu Y, Yu T, Ong C-K, Shen Z, Wee A T-S and Sow C-H 2007 Tailoring wettability change on aligned and patterned carbon nanotube films for selective assembly J. Phys. Chem. B 111 1672-8

[20] Aria A I 2013 Control of Wettability of Carbon Nanotube Array by Reversible Dry Oxidation for Superhydrophobic Coating and Supercapacitor Applications 2013

[21] Lei Y, Gao G, Liu W, Liu T and Yin Y 2014 Synthesis of silver nanoparticles on surface-functionalized multi-walled carbon nanotubes by ultraviolet initiated photo-reduction method Appl. Surf. Sci. 317 49-55

[22] Gururaj M and Neelgund A O 2011 Deposition of silver nanoparticles on dendrimer functionalized multiwalled carbon nanotubes: synthesis, characterization and

antimicrobial activity J. Nanosci Nanotechnol. 11 3621-9

[23] Singh P, Lamanna G, Ménard-Moyon C, Toma F M, Magnano E, Bondino F, Prato M, Verma S and Bianco A 2011 Formation of efficient catalytic silver nanoparticles on carbon nanotubes by adenine functionalization Angew. Chemie-Int. Ed. 50, 9893-7

[24] Yang J, Zhang Z, Men X, Xu X and Zhu X 2010 Reversible superhydrophobicity to superhydrophilicity switching of a carbon nanotube film via alternation of UV irradiation and dark storage Langmuir 26 10198-202

[25] Karumuri A K, He L and Mukhopadhyay S M 2015 Tuning the surface wettability of carbon nanotube carpets in multiscale hierarchical solids Appl. Surf. Sci. 327 122-30

[26] Rodriguez-Gattorno G and Diaz D 2002 Metallic nanoparticles from spontaneous reduction of silver (I) in DMSO. Interaction between nitric oxide and silver nanoparticles J. Phys. Chem. B 106 2482-7

[27] Maleszweski A 2004 The funtionalization and characterization of adherent CNT with Ag NP for biological applications Chem. ...

[28] Barney I T 2012 Fabrication and testing of hierarchical carbon nanostructures for multifunctional applications

[29] Dal Lago V, França de Oliveira L, de Almeida Gonçalves K Kobarg J and Borba Cardoso M 2011 Size-selective silver nanoparticles: future of biomedical devices with enhanced bactericidal properties J. Mater. Chem. 2112267

[30] Pal S, Tak Y K and Song J M 2007 Does the antibacterial activity of silver nanoparticles depend on the shape of the nanoparticle? A study of the Gram-negative bacterium Escherichia coli Appl. Environ. Microbiol. 73 1712-20

[31] Bondarenko O, Ivask A, Käkinen A, Kurvet I and Kahru A 2013 Particle-cell contact enhances antibacterial activity of silver nanoparticles PLoS One 8 e64060 


\section{QUERY FORM}

JOURNAL: Nanotechnology

Author: A K Karumuri et al

TiтLE: Silver nanoparticles supported on carbon nanotube carpets: influence of surface functionalization Article ID: nanoaa1670

The layout of this article has not yet been finalized. Therefore this proof may contain columns that are not fully balanced/ matched or overlapping text in inline equations; these issues will be resolved once the final corrections have been incorporated.

\section{SQ1}

Please be aware that the colour figures in this article will only appear in colour in the online version. If you require colour in the printed journal and have not previously arranged it, please contact the Production Editor now.

We have been provided funding information for this article as below. Please confirm whether this information is correct.

Division of Chemical, Bioengineering, Environmental, and Transport Systems: 1449582.

\section{Page 1}

Q1

Section headings have been ordered sequentially as per the journal style. Please check and approve.

Page 11

Q2

Please check the details for any journal references that do not have a link as they may contain some incorrect information.

\section{Page 11}

Q3

Please provide the surname for the author [S M M] in reference [8].

\section{Page 11}

Q4

Please provide the volume and page number or article number in references [8 and 27].

Page 11

Q5

Please provide the page range or article number in reference [10].

\section{Page 12}

Q6

Please check if this reference is to a book, journal conference proceedings or other and supply the complete details as appropriate.

\section{Page 12}

Q7

Please check if this reference is to a book, journal conference proceedings or other and supply the complete details as appropriate. 